\title{
Agieren deutsche Medien gemäß dem Propagandamodell? Das Beispiel Bombardierung von Krankenhäusern durch die USA und Russland
}

Kim Kristin Mauch

Keywords: Doppelte Standards, Propagandamodell, Kriegsberichterstattung, Qualitative Inhaltsanalyse

\section{Abstract}

Häufig wird der Vorwurf laut, die Medien würden in Bezug auf die Vereinigten Staaten und Russland mit zweierlei Maß messen. Hier wird eine qualitative Inhaltsanalyse durchgeführt, um zu überprüfen, ob Nachrichten in deutschen Leitmedien tatsächlich ideologisch gefärbt sind. Als theoretischer Hintergrund dient das Propagandamodell von Herman und Chomsky. Es werden zu zwei ähnlichen Vorfällen - die Bombardierung eines Krankenhauses in Afghanistan durch die USA 2015 und die Bombardierung eines Krankenhauses in Syrien durch Russland 2016 - werden dafür Artikel aus überregionalen Tageszeitungen und TV-Nachrichtensendungen ausgewertet. Die These, dass die Opfer von möglichen Kriegsverbrechen unterschiedlicher Konfliktparteien unterschiedlich dargestellt werden („wertvolle“ vs. „wertlose Opfer"), ließ sich nur in Teilen bestätigen. Bestätigt wurde jedoch, dass die USA und Russland als Verantwortliche für die Verbrechen in den Medien unterschiedlich beurteilt wurden.

Mauch, Kim Kristin. 2019. „Agieren deutsche Medien gemäß dem Propagandamodell? Das Beispiel Bombardierung von Krankenhäusern durch die USA und Russland“. In Ideologie, Kritik, Öffentlichkeit. Verhandlungen des Netzwerks Kritische Kommunikationswissenschaft, herausgegeben von Uwe Krüger und Sebastian Sevignani, 254-268. Universität Leipzig. DOI: 10.36730/ideologiekritik.2019.12

Kim Kristin Mauch | Universität Leipzig | kmkrstnmch@gmail.com 


\section{Einleitung}

Viele Menschen haben hohe Erwartungen an die Medien: Sie sollen ohne große Zeitverzögerungen und immer wahrheitsgetreu berichten. Vor allem letzteres ist ihnen besonders wichtig. Doch häufig widersprechen sich unterschiedliche Publikationen oder interpretieren die Ereignisse auf unterschiedliche Weise. Während eine Vielfalt der Meinungen wünschenswert und als Ausdruck von Demokratie verstanden werden kann, ist eine Vielfalt der Wahrheiten eher bedenklich.

Im Rahmen einer Bachelorarbeit habe ich an der Universität Leipzig eine qualitative Inhaltsanalyse durchgeführt, deren Ziel es war, exemplarisch zu überprüfen, ob Nachrichten in deutschen Medien ideologisch gefärbt sind. Als ideologietheoretischer Hintergrund diente dabei das Propagandamodell von Edward S. Herman und Noam Chomsky.

\section{Das Propagandamodell}

Das Propagandamodell entwickelten Herman und Chomsky in den 1980er Jahren nach langjährigen Studien zur Arbeitsweise der Medien. Dahinter steht der Grundgedanke, dass das amerikanische Mediensystem den Spielregeln der freien Marktwirtschaft unterliegt und deshalb nicht gänzlich unabhängig berichten kann. Ein potenzielles Bias entsteht beispielsweise bei der Nachrichtenauswahl, aber auch schon bei der Auswahl der Journalisten, die Nachrichten verbreiten. Da viele Journalisten davon überzeugt sind, durch Berufsethik und professionelle Nachrichtenfaktoren objektiv und unabhängig zu berichten, stellen Herman und Chomsky die These auf, dass auch unbewusste Selbstzensur im System eine Rolle spielt. In dem Buch Manufacturing Consent vergleichen sie die Berichterstattung von US-Medien zu Menschenrechtsverletzungen und kommen zu dem Schluss: „In sum, a propaganda approach to media coverage suggests a systematic and highly political dichotomization in news coverage based on serviceability to important domestic power interests" (Herman und Chomsky 1994, 35). 
Ihnen zufolge lassen sich fünf Filter identifizieren, die den Informationsfluss in der Regel auf die Inhalte reduzieren, die mit der aktuellen Meinungsspanne unter den politischen und wirtschaftlichen Eliten kompatibel sind.

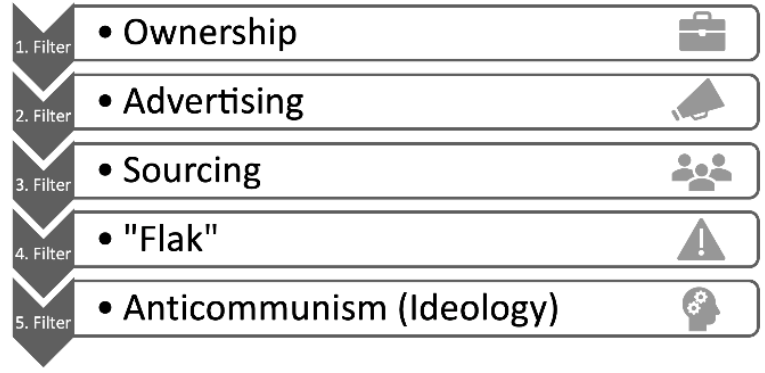

Abb. 1: Die fünf Filter des Propagandamodells

Der erste Filter bezieht sich auf die Interessen der Medieneigentümer. Zum Teil halten einzelne, sehr vermögende Familien eine Mehrheit der Anteile an Medienunternehmen, doch häufig gehören Medien auch einer Vielzahl von Investoren. Beide Gruppen sind in der Regel auch mit anderen Wirtschaftszweigen eng verknüpft. Sie haben deshalb gemeinsame Interessen mit der Wirtschaftselite. Das größte gemeinsame Interesse ist der Profit (ebd., 3-14).

Der zweite Filter besagt, dass Werbung eine gestaltende Wirkung auf die Medienlandschaft hat. In der Marktwirtschaft stehen Zeitungen, Fernsehsender etc. in Konkurrenz zueinander. Diejenigen, die sich zusätzlich über Werbung finanzieren und nicht ausschließlich über die Einnahmen aus dem Verkauf, können ihre Zeitungen günstiger anbieten und so die Reichweite steigern. Um attraktiv für Anzeigen zu sein, müssen die Zeitungen mit ihren Inhalten jedoch ein gutes Umfeld für Werbung bieten (ebd., 14-18).

Der dritte Filter betrifft die Quellen der Berichterstattung nutzen. Journalist*innen sind $\mathrm{zu}$ einem großen Teil auf Nachrichtenagenturen und auf die Öffentlichkeitsarbeit ressourcenstarker Organisationen angewiesen, da jeden Tag eine ausreichende Menge an Material zur Verfügung stehen muss und eigenständige Recherche arbeits- und kostenaufwendig ist. Viele der Informationen werden von Regierungsinstitutionen oder 
Unternehmen bereitgestellt. Oft sind diese Organisationen auch der einzige Partner, an den sich Journalisten für Informationen wenden können. Die Materialien sind in der Regel an die Bedürfnisse der Journalisten angepasst und werden in der Regel als zuverlässig eingeschätzt. Die Hürde, die diese Art von Informationen überwinden muss, um einen Weg in die Medien zu finden, ist deshalb sehr gering. Dies bedeutet gleichzeitig, dass es Organisationen oder auch Privatpersonen, die im Vergleich mit weniger Ressourcen und Reputation ausgestattet sind, wesentlich schwerer haben, ihre Informationen und ihre Meinung in den Medien zu verbreiten (ebd., 18-26).

Mit dem vierten Filter "Flak" beschreiben Herman und Chomsky Reaktionen von Staat und Konzernen auf unliebsame Informationen und Meinungen. In Form von direkter oder indirekter Rückmeldung wird Druck ausgeübt, so dass dem Medienunternehmen Kosten und Aufwand entstehen. Unterschiedliche Produzenten von Flak verstärken einander mit ihrer Kritik an der Berichterstattung. Manche Organisationen und Think Tanks wurden eigens zu dem Zweck gegründet, Flak zu produzieren (ebd., 26-31).

Der fünfte Filter nennt sich „Antikommunismus als Kontrollmechanismus“. Er wird als Ideologie benutzt, mit der die Bevölkerung auf einen gemeinsamen Feind eingeschworen werden soll. Da das Konzept „Kommunismus" jedoch nicht klar definiert ist, kann es gegen jeden verwendet werden, der Besitzinteressen bedroht oder mit Feinden der Regierung sympathisiert. Diesem Filter wohnt inne, dass er Themen in ein dichotomes Framing einbettet, das die Welt in Freunde und Feinde unterteilt (ebd., 31- 35).

Nach Herman und Chomsky hat die Wirkungsweise der Filter zur Folge, dass die Medien in ihrer Berichterstattung mit zweierlei Maß messen. Beachtung finden demnach vor allem Themen, die den Interessen der Elite dienlich sind. In der außenpolitischen Berichterstattung wird zwischen "worthy victims" und "unworthy victims" unterschieden. Als „wertvoll“ werden diejenigen Opfer dargestellt, die der Feind verschuldet hat, da sie sich für propagandistische Zwecke nutzen lassen. „Wertlos“ erscheinen dagegen die Opfer, die selbst oder von befreundeten Staaten verursacht wurden (ebd., 42ff.). Es werden große Unterschiede in den Medienbeiträgen über „worthy“ und „unworthy victims“ erwartet, sowohl in 
quantitativer als auch in qualitativ-inhaltlicher Hinsicht. Im Fall von „unworthy victims" werden staatliche Quellen massiv und unkritisch benutzt. Es wird davon ausgegangen, dass der eigene Staat für Frieden, Demokratie und Wahrheit steht. Im Fall von „worthy victims“ geht man davon aus, dass der feindliche Staat das Gegenteil verkörpert. Außerdem werden große investigative Recherchen unternommen, um das Unrecht in feindlichen Staaten aufzudecken und hohe Amtsträger für den Missbrauch von Macht verantwortlich zu machen. Diese Anstrengungen werden jedoch nicht im eigenen Land oder bei Bündnispartnern unternommen. Des Weiteren werden Unterschiede in Umfang, Platzierung und Sprache der Beiträge vermutet (ebd., 35; Chomsky 2003, 202).

An diesem Modell lässt sich in einigen Punkten Kritik üben. So lässt sich beispielsweise nicht bestätigen, dass die Elite tatsächlich einheitliche Interessen vertritt (Klaehn 2002, 154). Außerdem stammen die Erkenntnisse Hermans und Chomskys hauptsächlich aus Analysen der außenpolitischen Berichterstattung, wo in den meisten Fällen der Zugang zu Quellen für Journalist*innen wesentlich schwieriger ist als im Inland (Lang und Lang 2004, 93-100). Am wichtigsten ist jedoch die Erkenntnis, dass sich durch die Filter zwar bestimmte Muster vorhersagen lassen, doch belegt ein Aufzeigen dieser Muster keineswegs die Existenz bzw. Wirkung der spezifischen Filter. Die Prozesse, wie es zu dem Bias kommt, sind nicht belegbar (Corner 2003, 369; Thompson 2009, 74; Krüger 2019, 67). Deswegen bietet das Propagandamodell zwar einen nützlichen Rahmen für eine kritische Betrachtung der Nachrichtenauswahl, es fehlt jedoch an empirischen Methoden, die Schlüsse auf die Wirkungsweise der Filter zulassen.

\section{Methodisches Vorgehen}

Mit einer Inhaltsanalyse soll die Frage geklärt werden, ob sich die von Herman und Chomsky angenommene Dichotomisierung von "wertvollen" und "wertlosen Opfern" auch in deutschen Leitmedien feststellen lässt. Verglichen wird die Berichterstattung über zwei Fälle der Bombardierung eines Krankenhauses.

In Afghanistan wurde in den frühen Morgenstunden des 3. Oktober 2015 ein Krankenhaus der internationalen Organisation Ärzte ohne Gren- 
zen in der Stadt Kundus durch mehrere Raketen getroffen. Die Hilfsorganisation sprach von mehreren gezielten Treffern auf das Hauptgebäude, in dem sich Intensivstation und Notaufnahme befanden, und von mindestens 42 Toten, darunter 14 Mitarbeitern und 24 Patienten. Auch drei Kinder sollen bei dem Angriff ums Leben gekommen sein (MSF 2015).

Ein sehr ähnlicher Vorfall ereignete sich in Syrien am 15. Februar 2016. Ein von Ärzte ohne Grenzen unterstütztes Krankenhaus in der Stadt Ma'arat Al Numan in der syrischen Provinz Idlib wurde am Morgen durch einen Luftangriff komplett zerstört. Mindestens 25 Menschen starben, davon gehörten neun zum Personal, 16 Personen waren Patienten. Weitere zehn Menschen wurden schwer verwundet. Ein nahegelegenes Krankenhaus, das die Verwundeten versorgte, wurde laut Ärzte ohne Grenzen nur eine Stunde später ebenfalls zum Ziel (MSF 2016).

Für das Bombardement in Afghanistan wurde die US-amerikanische Luftwaffe verantwortlich gemacht. Der Luftangriff in Syrien wird der Koalition der syrischen Regierung zugeschrieben, an der auch Russland beteiligt ist.

Damit folgt diese Arbeit dem Beispiel von Herman und Chomsky (1994), die in Manufacturing Consent die US-Medien auf unterschiedliche Darstellungen von Menschenrechtsverletzungen in El Salvador, Guatemala, Nicaragua, Vietnam, Kambodscha und Laos untersuchten. Bei der Auswahl der Fälle stand im Vordergrund, dass eine möglichst große Ähnlichkeit zwischen den Ereignissen besteht. Außerdem sollte der zeitliche Abstand gering sein, um ein ähnliches geopolitisches Umfeld und Meinungsklima zu gewährleisten. Ein Paarvergleich wie der hier vorliegende ist deshalb interessant, weil sich die die USA und Russland als geopolitische Antagonisten gegenüberstehen und Deutschland im Rahmen der Nato eng an die USA gebunden ist (bzw. damals, vor der Ära Trump, noch war). Die Frage ist also, ob die Berichterstattung deutscher Medien über die beiden sehr ähnlichen Vorfälle von dieser geopolitischen Konstellation beeinflusst ist. In beiden Konflikten war auch Deutschland zu dem betreffenden Zeitpunkt militärisch in den Ländern aktiv, wenn auch nicht an den Angriffen beteiligt.

Zur Analyse wurden 24 Artikel aus sieben überregionalen Zeitungen und 14 Filmbeiträge aus vier TV-Nachrichtensendungen über diese beiden Vorfälle herangezogen. Für das Sample wurden sowohl Leitmedien 
bzw. reichweitenstarke Medien als auch einige alternative Blätter ausgewählt, um ein möglichst breites Spektrum der Medienlandschaft in Deutschland abzubilden. Im Fernsehbereich wurden die reichenwertenstärksten Hauptnachrichtensendungen des öffentlich-rechtlichen und privaten Fernsehens ausgewählt: die ARD-Tagesschau (20.00-Uhr-Ausgabe), ZDF heute (19.00 Uhr), RTL Aktuell (18.45 Uhr) und Sat.1 Nachrichten (19.55 Uhr). Als überregionale Tageszeitungen wurden die Bild-Zeitung, die Welt, die Frankfurter Allgemeine Zeitung, die Süddeutsche Zeitung, die taz, das Neue Deutschland und die Junge Welt ausgewählt. Die hier gewählte Reihenfolge entspricht der angenommenen redaktionellen Linie der Blätter im politischen Spektrum von rechts nach links. Die Bild ist dabei im Gegensatz zu den Qualitätsblättern als Boulevardzeitung einzustufen. Wegen ihrer enormen Reichweite von 9,9 Mio. Lesern ist sie in der deutschen Medienlandschaft wichtig (Medien-Analyse agma, 2018). Die Junge Welt und das Neue Deutschland als kleinere linke Tageszeitungen dienen als Kontrollgruppe, um eventuelle Unterschiede zu den „Mainstream-Medien" zu erkennen.

Der Untersuchungszeitraum wurde eingegrenzt auf den jeweiligen Tag des Ereignisses sowie die drei Folgetage. Es wurden nur Beiträge ausgewählt, in denen die Bombardierung der Krankenhäuser explizit erwähnt wurde. Bei den TV-Beiträgen wurde aus forschungsökonomischen Gründen wurde nur der sprachliche Inhalt analysiert. Die Textpassagen wurden mit Hilfe von MAXQDA kodiert.

Die Kategorien leiten sich aus dem Propagandamodell ab. Um die These der „wertvollen“ und „wertlosen Opfer“ differenziert zu überprüfen, bildeten zwei Dimensionen die Grundlage des Kategoriensystem. Die eine basierte auf den Vorhersagen zu Vorfällen, für die Staaten verantwortlich sind, die keine enge partnerschaftliche Beziehung zu Deutschland haben. Merkmale dafür waren Berichte an prominenter Stelle, in dramatischem Stil und mit vielen menschlichen Attributen der Opfer, Empörung, der Wunsch nach Aufklärung und Sanktionen sowie die geringe Verwendung staatlicher Quellen aus dem verantwortlichen „Feindstaat“. Die zweite Dimension basiert auf den Vorhersagen zu tödlichen Vorfällen, für die Staaten verantwortlich sind, zu denen Deutschland enge partnerschaftliche Beziehungen pflegt. Kategorien hierfür waren die starke und unkritische Verwendung staatlicher Quellen aus dem befreundeten Staat, nur wenig Detailbeschreibung, minimale Vermenschlichung der 
Opfer und wenig Kontext. Daneben wurden weitere Merkmale in Sachen Platzierung, Überschriftengebung und Wortgebrauch kodiert.

\section{Ergebnisse}

Insgesamt fanden sich 24 Zeitungsartikel (davon 6 in der Jungen Welt und im Neuen Deutschland) und 14 TV-Beiträge zu den Krankenhaus-Bombardierungen. Es wurden insgesamt 662 Textstellen codiert. Für alle Kategorien, die Merkmale für „wertvolle Opfer" darstellen, wurden mehr Codings in den Artikeln über Syrien gefunden, während es mehr Codings in den Kategorien für "wertlose Opfer" im Fall von Afghanistan gab. Dies bestätigt zunächst die These von Herman und Chomsky. Die Bestätigung ist noch eindeutiger, betrachtet man die Zahlen für die Mainstream-Medien gesondert. Bezieht man Junge Welt und Neues Deutschland also nicht mit ein, sind die Unterschiede in der Darstellung der Opfer in Syrien und Afghanistan noch größer (siehe Tab. 1).

Tab. 1: Anzahl der Codings nach Art der Medien

\begin{tabular}{|c|c|c|}
\hline & Afghanistan & Syrien \\
\hline \multicolumn{3}{|c|}{ Merkmale für „wertvolle Opfer" } \\
\hline $\begin{array}{r}\text { Anzahl der Codings } \\
\text { insgesamt }\end{array}$ & 187 & 258 \\
\hline $\begin{array}{l}\text { Anzahl der Codings } \\
\text { ohne ND und JW }\end{array}$ & 100 & 242 \\
\hline \multicolumn{3}{|c|}{ Merkmale für „wertlose Opfer" } \\
\hline $\begin{array}{r}\text { Anzahl der Codings } \\
\text { insgesamt }\end{array}$ & 110 & 50 \\
\hline $\begin{array}{l}\text { Anzahl der Codings } \\
\text { ohne ND und JW }\end{array}$ & 102 & 45 \\
\hline
\end{tabular}


Weil die Anzahl der Codings allein nicht aussagekräftig genug ist, soll das Material im Folgenden möglichst dicht beschrieben werden. Eingegangen wird auf die Position der Beiträge, den Umfang, die Überschriften, die Art der Sprache, die Auswahl der Quellen, die vermittelte Einstellung zum Staat, die Absicht und die zugeschriebene Verantwortung.

\subsection{Position}

Über den Vorfall in Syrien mit russischer Verantwortung wurde häufiger an prominenter Stelle (das heißt auf den ersten drei Seiten oder unter den ersten drei Beiträgen einer Sendung) berichtet als über den Vorfall in Afghanistan mit US-amerikanischer Verantwortung. Der Unterschied fällt bei den Tageszeitungen geringer aus als bei den TV-Sendungen.

\subsection{Umfang}

Die Analyse ergab, dass die Zeitungen (ohne $J W$ und ND) über den Vorfall in Syrien mit durchschnittlich 283 Wörtern pro Artikel berichteten, über den Vorfall in Afghanistan mit durchschnittlich 307 Wörtern pro Artikel, was der Annahme von Herman und Chomsky zum Umfang widerspricht. Die TV-Beiträge zu Syrien waren im Durchschnitt 1 Minute und 21 Sekunden lang, die TV-Beiträge zu Afghanistan waren im Durchschnitt nur 45 Sekunden lang, was das Propagandamodell bestätigt.

\section{3 Überschriften}

Eine Analyse der Überschriften der Zeitungsartikel ergab, dass in beiden Fällen ähnlich häufig Überschriften gewählt wurden, die sich konkret auf die Bombardierungen, die Opfer oder Kriegsverbrechen beziehen und damit als Anzeichen für „,wertvolle Opfer" gelten. Dies widerspricht also der Annahme, dass es hier deutliche Unterschiede gibt. Am ehesten entspricht dem Propagandamodell die FAZ, die zum Vorfall in Afghanistan am 5.10.2015 titelte "Gouverneur: Krankenhaus war eine Taliban-Stellung“ und am Folgetag "Afghanen forderten Luftschlag an“. Diese Überschriften ordnen die Bombardierung also als vernünftig und nachvollziehbar bzw. als bedauerlichen Fehler ein, lenken von den Opfern eher ab und mindern die Verantwortung der US Air Force. 


\subsection{Art der Sprache}

Die Analyse der Sprache bezog sich auf die Einstufung der Ereignisse als Kriegsverbrechen, auf Details zu den Angriffen, die medizinische Versorgungslage und die Todesfälle sowie auf die Erwähnung von Kindern, Vermissten oder Verletzten.

Bild, Welt, ZDF, Sat.1 und RTL berichteten tendenziell auffälliger über die syrischen Opfer, während FAZ, Junge Welt und Neues Deutschland die afghanischen Opfer detaillierter beschrieben. Hier verhält sich also die konservative FAZ ähnlich wie die linken Zeitungen und entgegen der Annahmen des Propagandamodells. Die Verwendung der Sprache bei SZ, taz und $A R D$ war hingegen bei beiden Vorfällen sehr ähnlich. Diese Ausgewogenheit widerspricht ebenfalls den Annahmen von Herman und Chomsky.

\subsection{Auswahl der Quellen}

In allen Medienbeiträgen der Mainstream-Medien (also ohne JW und ND) zur Bombardierung in Syrien wurde insgesamt 38-mal eine nicht-staatliche Quelle direkt oder indirekt zitiert, davon 18-mal die Organisation Ärzte ohne Grenzen (aber auch vier weitere NGOs). Neunmal wurden oppositionsnahe syrische Organisationen zitiert. In der Afghanistan-Berichterstattung wurden nur 22 nicht-staatliche Quellen zitiert, davon allein 19mal Ärzte ohne Grenzen. Außer dieser Organisation wurden nur an drei Stellen Mitarbeiter des Krankenhauses und zwei Mal Augenzeugen zitiert. Die Vielfalt der Quellen im Fall von Syrien war also größer, was den Annahmen des Propagandamodells entspricht.

Für die Berichterstattung zu Syrien wurden in den Mainstream-Medien 47 staatliche Quellen zitiert. Von diesen Äußerungen wurden 25 von Quellen getätigt, die sich westlichen bzw. Nato-Staaten zuordnen lassen (Deutschland, USA, Türkei, Frankreich) und die starke Kritik an Russland üben. Außerdem wurde zweimal die syrische Opposition zitiert, die sich genau wie die westlichen Quellen gegen Russland und Syrien aussprach. Im Gegensatz dazu kamen 36-mal staatliche Quellen zum Vorfall in Afghanistan zu Wort. In 24 Fällen äußerten sich die US-Regierung oder das Militär selbst zu dem Vorfall, der ihnen angelastet wurde. In weiteren neun Fällen wurde die afghanische Regierung zitiert, die mit den USA zusammenarbeitete. Nur dreimal kamen westliche Quellen zu Wort, die 
eine Untersuchung und Aufklärung des Vorfalls fordern (aus Frankreich und Deutschland). Stimmen aus dem nicht-westlichen geopolitischen Spektrum gab es keine.

Die generelle Vorliebe für staatliche Quellen des Westens, die Neigung zu NGOs und Anti-Assad-Gruppen im Fall Syrien und die häufige Zitation von US-Regierung und US-Militär im Fall Afghanistan bestätigen die Annahmen des Propagandamodells.

\subsection{Einstellung zu den verantwortlichen Staaten}

In der Mainstream-Berichterstattung über Syrien und Russland fanden sich 35 Hinweise, dass die Annahmen aus dem Propagandamodell über die Einstellung $\mathrm{zu}$ geopolitischen Gegnern stimmen. Mehrere Mainstream-Medien (aber auch das Neue Deutschland) stellten Syrien und Russland als Verursacher von Gewalt und Krieg dar. In einigen Textstellen wird beschrieben, wie Russland und Syrien Terrorismus begünstigen. So zitiert etwa die FAZ am 16.2.2016 den türkischen Ministerpräsidenten: „Davutoglu sagte bei einem Besuch in Kiew, Russland sei ebenso wie die Terrororganisation ,Islamischer Staat' (IS) verantwortlich für Verbrechen gegen die Menschlichkeit, indem es ,barbarische Angriffe auf die friedliche Bevölkerung' ausführe."

Ganz im Gegensatz dazu steht die Darstellung von westlichen Ländern in der Syrien-Berichterstattung, genau genommen Deutschland und der USA. Sie wurden als die Kräfte beschrieben, die den Frieden nach Syrien bringen wollen. Häufig wurde über Angela Merkel berichtet, die sich für eine Flugverbotszone aussprach, um die einheimische Bevölkerung zu schützen. Den USA wurde in der FAZ um 6.10.2015 Platz eingeräumt für ihre Darstellung der Ereignisse in Afghanistan: „Daraufhin sei ein Luftangriff geflogen worden, um die Bedrohung durch die Taliban , auszuschalten'. Dabei seien ,mehrere Zivilisten aus Versehen getroffen' worden, sagte General Campbell weiter und sprach den Angehörigen der Opfer sein ,tiefstes Mitgefühl' aus." Allerdings wurde dieses Zitat anschließend eingeordnet: „Bislang hatte das amerikanische Militär erklärt, dass ihre eigenen Truppen unter Beschuss geraten und Luftunterstützung angefordert hätten." Aus diesen Ungereimtheiten eine Schlussfolgerung zu ziehen, wird allerdings den Leser*innen selbst überlassen.

Es gab in der Afghanistan-Berichterstattung Hinweise darauf, dass Gewalt und Krieg auch von den USA ausgehen. An anderer Stelle wurden 
die Vereinigten Staaten der Lüge bezichtigt. Manchmal geht diese Schlussfolgerung aber nur implizit aus der Gegenüberstellung der Version des US-Militärs mit der Darstellung von Ärzte ohne Grenzen hervor. Es fiel jedoch auf, dass nur in neun Textstellen negativ über Amerika berichtet wurde. In den alternativen Medien $(J W$ und $N D)$ finden sich hingegen 20 US-kritische Textstellen.

Dass diese Situation für deutsche Medien eher ungewohnt ist, verdeutlichte ein Artikel der Welt. Hier hieß es am 5.10.2015: „Der Angriff in Kundus überschattet Washingtons Kritik an russischen Militäraktionen in Syrien [...] Die Bombardierung des Hospitals in Kundus verschiebt in dieser Debatte auf einen Schlag die moralischen Gewichte." Dagegen weisen mehrere Medien zu Gunsten der USA darauf hin, dass sie gemeinsam mit Afghanistan und der Nato den Terror der Taliban bekämpfen.

\subsection{Absicht}

Der russisch-syrischen Koalition wurde an vielen Stellen böse Absicht unterstellt. Die Vorwürfe lauteten, es habe sich um einen gezielten Angriff gehandelt und es sei eine Strategie gewesen, die Zivilbevölkerung anzugreifen. Außerdem wurde berichtet, es gebe einen Trend dahingehend, Gesundheitseinrichtungen zu zerstören, und die Gewalt würde trotz der angekündigten Feuerpause zunehmen. So heißt es etwa in der Welt vom 16.2.2016 „Der Marburger Bund verurteilt die Anschläge. ,Die fortwährenden Angriffe auf Krankenhäuser in Syrien sind abscheuliche Verbrechen an der Zivilbevölkerung', sagte der Verbandsvorsitzende Rudolf Henke." Insgesamt 23 Textstellen in dem Material der Mainstream-Medien lassen auf böse Absicht bzw. Vorsatz schließen.

Im Fall Afghanistan berichteten nur die alternativen Medien (Junge Welt und Neues Deutschland) sowie die taz und die FAZ über einen gezielten beziehungsweise beabsichtigten Angriff. Die anderen Medien berichten lediglich über die Darstellung von MSF, nach der die Lage des Krankenhauses an das US-Militär und andere Institutionen übermittelt worden war, sich keine Taliban-Kämpfer in der Klinik befanden und trotzdem der Angriff fortgesetzt wurde. Aus diesen Hinweisen konnten die Leser*innen eigene Schlüsse ziehen. Zusammengefasst ließ sich aus zehn Textstellen eine mögliche Absicht ableiten. Allerdings ließen 28 Textstellen auf 
eine andere Darstellung schließen. Mehrere Medien zitierten US-Präsident Barack Obama, der im Zusammenhang mit den Angriffen von einer "Tragödie" oder einem "tragischen Vorfall" sprach. Außerdem wurde insgesamt 22-mal über die Äußerungen des US-Militärs und der afghanischen Armee berichtet, es hätten sich Taliban in der Klinik befunden, die US-Truppen angegriffen hätten.

\subsection{Verantwortung}

In der Frage der Verantwortung für die Vorfälle zeigte sich, dass in der Syrien-Berichterstattung alle Medien Quellen nannten, die die Verantwortung bei Syrien und/oder Russland sehen. Besonders Russland wird 21mal explizit als möglicher Verursacher genannt. Vier Medien veröffentlichten auch eine Stellungnahme des syrischen Botschafters in Moskau, der die USA für verantwortlich hielt. Andere berichteten, dass bislang noch nicht abschließend geklärt werden konnte, wer verantwortlich sei. Sechs Medien (FAZ, taz, ND, ARD, Sat.1, ZDF) erwähnten kurz, dass Russland die Vorwürfe zurückweist.

Über Afghanistan berichteten sechs Medien an insgesamt 14 Stellen, dass die USA für den Angriff auf das Krankenhaus verantwortlich seien. Die Medien Welt, SZ, ARD und Sat.1 berichteten allerdings, es habe sich in Afghanistan offensichtlich um ein Versehen des US-Militärs gehandelt, obwohl die Schilderungen von Ärzte ohne Grenzen einen anderen Schluss nahelegen. So heißt es in der SZ vom 5.10.2015 schon in der Überschrift „Obama entschuldigt sich für Bomben auf Klinik - Offenbar irrtümlicherweise griff die US-Armee das Krankenhaus in Kundus an, in dem sich angeblich Taliban verschanzt hatten." Zwei Medien äußerten jedoch, dass die Umstände noch unklar seien. Die SZ berichtete an den Folgetagen, dass ein Irrtum unwahrscheinlich sei. Auffällig war jedoch, dass in der Afghanistan-Berichterstattung wesentlich häufiger nur zwischen den Zeilen zu finden war, wer die Schuld an dem Unglück trug, während die Verantwortung im Syrien-Fall explizit zugewiesen wurde. 


\section{Fazit}

Kann also von einer starken Dichotomisierung in den deutschen Medien gesprochen werden, bei der die Opfer "unserer" Gegner emporgehoben, während die selbst verschuldeten Toten verschwiegen werden? Zwar ließen sich Unterschiede in der Darstellung der Opfer von möglichen Kriegsverbrechen feststellen, doch kann nicht bestätigt werden, dass es zu einer Versächlichung oder Entmenschlichung durch die Medien im Fall von Afghanistan gekommen wäre. Die geringe Anzahl von Codings für die These der "wertlosen Opfer" deutet darauf hin, dass eher die Abwesenheit von Merkmalen für „,wertvolle Opfer" eine Rolle spielt. Die Opfer der Kriegsverbrechen in Afghanistan wurden also weniger häufig betont.

Ein weiteres Ergebnis ist, dass zwischen den untersuchten Fällen wichtige Unterschiede in der medialen Berichterstattung zu erkennen sind. Die Vielfalt der Quellen im Fall von Syrien weist darauf hin, dass hier größere Anstrengungen unternommen wurden, um Gesprächspartner zu finden, die die Menschenrechtsverletzungen kritisieren. Dass verstärkt auf offizielle Quellen aus der westlichen Welt zurückgegriffen wird, mag vielleicht an besseren Zugangsbedingungen liegen. Ebenfalls belegt werden konnte die Tendenz, Russland als Kriegstreiber darzustellen, während die USA und auch Deutschland als Friedensbringer oder Terrorismusbekämpfer daherkommen. Zwar berichten vier Medien über die USA als Verursacher von Gewalt, doch wird in ihrer Berichterstattung der Afghanistan-Vorfall als Irrtum oder Versehen beschrieben. Zusammenfassend lässt sich sagen, dass die Verantwortlichkeit im Fall Afghanistan weniger direkt angesprochen wurde als im Fall Syrien.

Damit stimmt die Arbeit überein mit den empirischen Forschungsergebnissen von Zollmann (2017, 211), der ebenfalls zu dem Schluss kommt, dass die Medien (hier: große Medien aus Deutschland, Großbritannien und den USA) kritischer über die Menschenrechtsverletzungen geopolitische Gegner berichten als über die eigenen bzw. die von geopolitischen Partnern. Einen möglichen Erklärungsansatz für die Erkenntnisse liefert Krüger (2017, 253-255). Dieser beschreibt, dass die meisten deutschen Journalisten mit westlichen Werten sozialisiert und deshalb von einer Nähe zur USA und der Nato geprägt sind. Die Arbeit entgegen der eige- 
nen Sozialisation und positiver Bilder über den Westen erfordere deswegen ein hohes Maß an Recherchearbeit und sei mit hohem Geld- und Zeitaufwand verbunden.

\section{Literatur}

Chomsky, Noam. 2003. Media Control. Wie die Medien uns manipulieren. Hamburg: Europa Verlag $\mathrm{GmbH}$.

Corner, John. 2003. „The Model in Question: A Response to Klaehn on Herman and Chomsky“. European Journal of Communication 18 (3): 367-375.

Herman, Edward. S., und Noam Chomsky. 1994. Manufacturing Consent: The Political Economy of the Mass Media. London: Vintage.

Klaehn, Jeffery. 2002. „A Critical Review and Assessment of Herman and Chomsky's ‘Propaganda Model'“. European Journal of Communication 17 (2): 147-182.

Krüger, Uwe. 2019. Meinungsmacht: der Einfluss von Eliten auf Leitmedien und Alpha-Journalisten: eine kritische Netzwerkanalyse. 2. Auflage. Köln: Herbert von Halem.

Krüger, Uwe. 2017. „Medien-Mainstream. Eine Streitrede wider Konformität im Journalismus und für eine kritische Journalistik". In Lügenpresse. Anatomie eines politischen Kampfbegriffs von Volker Lilienthal und Irene Neverla (248-265). Köln: Kiepenheuer \& Witsch.

Lang, Kurt, und Gladys Engel Lang. 2004. „Noam Chomsky and the Manufacture of Consent for American Foreign Policy“. Political Communication 21 (1): 93-101.

MSF. 2015. MSF demands explanations after deadly airstrikes hit hospital in Kunduz (Pressemitteilung). Kabul, Brüssel: Médecins Sans Frontières International. https:// www.msf.org/afghanistan-msf-demands-explanations-after-deadlyairstrikes-hit-hospital-kunduz. Zugegriffen: 16. November 2019.

MSF. 2016. At least 11 killed in another MSF-supported hospital attack in Idlib province (Pressemitteilung). Gaziantep: Médecins Sans Frontières International. https:// www.msf.org/syria-least-11-killed-another-msf-supported-hospital-attackidlib-province. Zugegriffen: 16. November 2019.

Thompson, Peter A. 2009. „Market Manipulation? Applying the Propaganda Model to Financial Media Reporting". Westminster Papers in Communication and Culture 6 (2): 73.

Zollmann, Florian. 2017. Media, propaganda and the politics of intervention. New York: Peter Lang.

\section{Open Access}

Dieser Beitrag erscheint unter der Creative-Commons-Lizenz CC-BY-SA 4.0: https://creativecommons.org/licenses/by-sa/4.0/ 